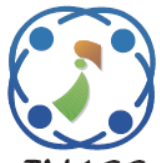

\title{
Delay Aware Hybrid Routing for Large Scale Cooperative D2D Networks
}

\section{Praveen Kumar Devulapalli ${ }^{1,2 *}$}

\author{
Pardha Saradhi Pokkunuri ${ }^{1}$
}

\author{
Maganti Sushanth Babu ${ }^{3}$
}

\author{
${ }^{1}$ Department of Electronics and Communication Engineering, \\ Koneru Laxmaiah Education Foundation, Guntur, Andhra Pradesh, India \\ ${ }^{2}$ Department of Electrical and Computer Engineering, \\ Vardhaman College of Engineering, Hyderabad, Telangana, India \\ ${ }^{3}$ Department of Electronics and Communication Engineering, \\ Matrusri Engineering College, Hyderabad, Telangana, India \\ * Corresponding author's Email: praveenkumar7.d@gmail.com
}

\begin{abstract}
Device-to-Device (D2D) communication can be better option for some applications like military and natural disaster places where we need temporary communication network. Selection of relay nodes and routing schemes are key issues in large scale Device-to-Device (D2D) cooperative networks which affects the performance of the network. The performance may be degraded because of wrong selection of relay nodes. In this paper, we proposed a hybrid cluster based D2D cooperative routing scheme by combining the geographic routing and clustering routing. The main idea of our algorithm is to establish communication between similar mobility devices to reduce the mobility effect since the link between the devices moving with the same (approximately) mobility is reliable. All the equal mobility devices are grouped to form a cluster; one of the devices in this is selected as a cluster head. We selected Cluster Head $(\mathrm{CH})$ based on geographic routing and threshold based cooperative communication is provided within the cluster to improve the network performance. We evaluated efficacy of our proposed scheme over conventional routing approaches discussed in the literature. From the results it was observed that the proposed routing scheme improves End-to-End transmission delay by 39\%, Energy consumption by 35\% (approximately) and Bit Error Rate performance.
\end{abstract}

Keywords: Large scale cooperative network, Routing, D2D communication, Clustering, Geographic routing.

\section{Introduction}

Cooperative communication is an efficient technique to mitigate the fading by providing the diversity using multiple single antenna transmitters and receivers. Further this technique is extended to High Frequency (HF) radio and large scale Mobile Ad hoc Networks (MANETs) to improve the network performance. Cooperative routing algorithms are cross layer approaches, formulated by combining the cooperative communication and routing at physical layer and network layer respectively $[1,2]$. Recently, cooperative communication is implemented in D2D communication, where one or more D2D nodes cooperates the source to improve the network performance via spatial diversity [3]. D2D communication has been included in 3GPP Release
12 as a key technology for $5 \mathrm{G}$ network to handle network capacity and/or coverage problems. In D2D communication, two nodes can communicate in ad hoc manner which results in reduced energy consumption and delay. Further single hop D2D communication (limited to specific geographic area) is extended to large scale networks (multi hop) to improve the network coverage area, quality of service (QoS), spectrum efficiency and high data rates [4].

Large scale D2D communication can be a better choice than the conventional cellular system in some applications like military and emergency search and rescue (natural disaster); where there is a need of establishing temporary communication network. In large-scale D2D communication networks, every intermediate nodes cooperates the source node by forwarding the information towards the destination. Hence, selection of relay nodes and routing are 
critical issues in large scale D2D communication [5, $6]$.

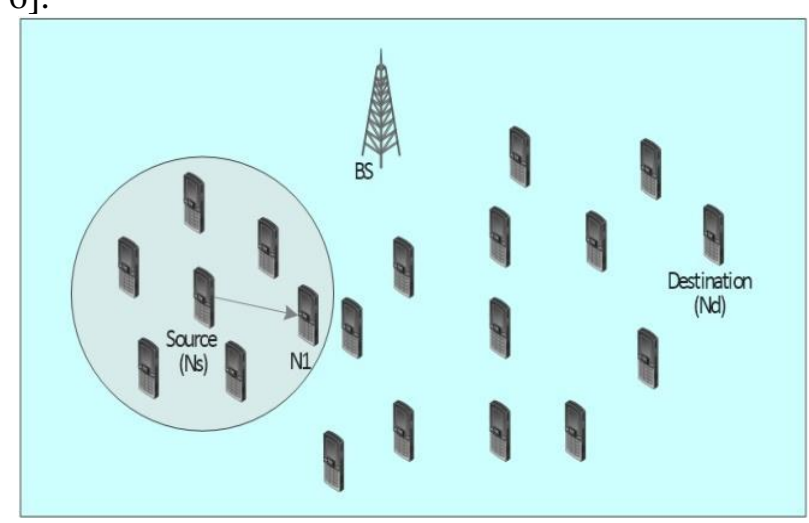

(a)

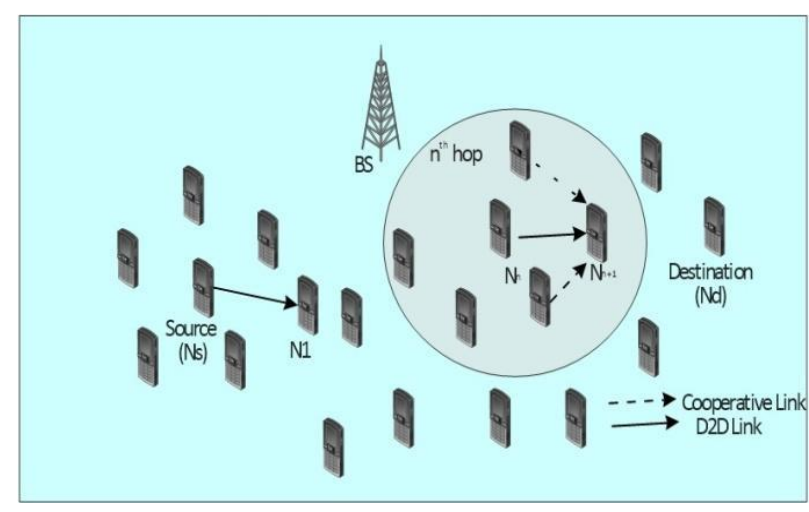

(b)

Figure 1: Large scale cooperative D2D network: (a) Hybrid cluster based D2D cooperative routing and (b) Intra-cluster D2D cooperative routing

In recent years, several routing algorithms have been proposed based on variety of parameters like: position of nodes, protocol operation, network structure, initiation of communication and path establishment or next hop selection [7-9]. The key challenges to be addressed while proposing routing algorithm are: network lifetime, energy consumption and forwarding efficiency. Clustering routing techniques addresses these challenges [10-12]. The Low Energy Adaptive Clustering Hierarchy $(\mathrm{LEACH})$ is one of the most popular clustering routing schemes. In recent years, many enhanced LEACH routing schemes have been proposed by focusing on modified cluster-heads $(\mathrm{CHs})$ selection, network topology and network expansion [13, 14]. But the main drawback in clustering routing is high end-to-end transmission delay.

Geographic routing (GR) is the one which attracts the researchers to use in network routing design because of its low complexity. GR scheme requires only the node positions to determine or select the relay nodes. The advantages of GR scheme are: (i) no need of channel information, (ii) scalability and (iii) low overhead information [15, 16]. Authors in [15] have proposed an Energy Efficient Cooperative Geographic Routing (ECGR), where in some set of nodes are determined at the network layer to form a cluster. Now, all the nodes in the cluster forward the information towards next cluster. But this routing scheme is effective in high density networks only. To minimize the energy consumption, a cooperative routing algorithm based on Quality of Service was presented in [17]. However, authors were not presented the affect mobility.

In [18], authors have been presented the performance analysis of non-regenerative wireless cooperative network over Rayleigh and Weibull fading channels with max-min best relay selection technique. The moment generating function (MGF) of signal-to-noise ratio (SNR) at the destination node was derived using the Weibull fading parameter. By using MGF, the lower bound value of symbol error rate and outage probability was determined. Analytical and simulation results concerning outage probability and symbol error rates are given under a varying number of relay nodes with a max-min relay selection technique to verify the accuracy of the derivation. However, in this relay selection technique; relay node is selected based on the link SNR which leads to high end-to-end transmission delay.

The authors in [19] develop an asymptotically optimal solution framework by transforming the original problem to an equivalent finite-horizon Markov decision process (MDP) with a fixed stage number. A novel approximation approach was proposed to address the curse of dimensionality, where the analytical expressions of approximate value functions are provided. Analytical bounds on the exact value function and approximation error are also derived. The approximate value functions depend on some system statistics, e.g., requesting users' distribution. One reinforcement learning algorithm was proposed for the scenario where these statistics are unknown. Furthermore, Energy consumption was a major issue in this research work.

An ad hoc on-demand distance vector (AODV) [20] is the typically well-known routing protocol belonging to ad hoc routing. This is designed as a loop-free, on-demand, single path, distance vector protocol, where a route discovery algorithm route reply messages were used to find a best path. As the extensions of on-demand routing, several routing protocols were proposed based on various criteria and design issues. For example, AODV-ETX [21] defined the link cost as the expected transmission times (ETX) to find an optimal routing path with the minimum total ETX, 
In [22], authors have been proposed a clusterbased cooperative packet transmission (CCPT) to improve the energy efficiency and end-to-end transmission delay. In this, authors designed inter cluster cooperative transmission based on successful reception of the receiving $\mathrm{CH}$ instead of receiving all the nodes in the cluster, which can enhance the robustness of transmission on the best path and effectively reduce the transmission times. The authors in [23] have proposed an opportunistic cooperative packet transmission (OCPT) scheme for multi hop cooperative network. In OCPT, before the transmission, a cluster head selects the transmitter and receivers to form MIMO. Because of multiple transmitters and receivers in every hop, end-to-end transmission time and energy consumption per node is considerably high.

Therefore, the energy consumption and shortest path routing is the main issue facing large-scale MANET which has to be sorted out. There are various techniques implemented but due to poor performance, reduced network lifetime, packet transmission problems, and high energy consumption still there is a lack in finding the solution. Hence a novel method is introduced to improve the end-to-end transmission delay and reduce energy consumption. In this paper, we have proposed a hybrid routing scheme for large scale cooperative D2D network, where in the selection of cluster head $(\mathrm{CH})$ is based on geographic position of the node and other nodes in the cluster which cooperates (if required) to the $\mathrm{CH}$ to forward the information. This hybrid routing technique is superior to the state-of-the-art routing schemes w.r.t. the end-to-end transmission delay and energy consumption.

The rest of the paper is organized as follows: description of the network given in section 2, routing algorithm, energy consumption, BER and end-to end transmission delay are presented in section 3. In section 4, we present the simulation results and we conclude in section 5 .

\section{System model}

We consider a large scale cooperative D2D network, which contains $S$ mobile nodes distributed randomly in $L \times L \mathrm{~m}^{2}$ area as shown in Fig. 1. Every node in the network is assumed to be self-organized and contains $K$ radio terminals with fixed transmit power $P$ and transmission coverage area $R$ Every node obtains its own location using GPS and list of neighbor nodes and their location by exchanging beacon signal for every $\alpha$ sec.

We assume that every node in the network measures link Signal to Noise Ratio (SNR) and
Table 1. Summary of notations

\begin{tabular}{|l|l|}
\hline Symbol & Description \\
\hline$S$ & Number of mobile nodes \\
\hline$P$ & Transmit Power \\
\hline$R$ & Transmission Coverage area \\
\hline$\alpha$ & Periodic interval (in sec) \\
\hline$S_{j}$ & Link Signal to Noise Ratio (SNR) \\
\hline$\Delta d_{i j}$ & Relative distance between node $i$ and $j$ \\
\hline$\Delta v_{i j}$ & Relative velocity \\
\hline$N_{S}$ & Source node \\
\hline$N_{d}$ & Destination node \\
\hline$G_{N_{S}}$ & $\begin{array}{l}\text { Set of nodes in transmission coverage } \\
\text { area of source node }\end{array}$ \\
\hline$\sigma^{2}$ & Noise Variance \\
\hline$h_{i j}$ & $\begin{array}{l}\text { Channel Coefficient between node } i \text { and } \\
j\end{array}$ \\
\hline$\delta_{i j}^{2}$ & variance between $i$ and $j$ \\
\hline$\tilde{X}$ & compressed encoded data \\
\hline$A_{N_{n-1}}$ & $n^{\text {th }}$ hop transmitter gain \\
\hline
\end{tabular}

relative velocity metrics based on received compressed beacon signal.

The link SNR between node $i$ and node $j$ is evaluated as

$$
\zeta_{j}=\frac{P\left|h_{i j}\right|^{2}}{\sigma_{j}^{2}}
$$

Based on the signal to noise ratio, node $i$ measures the relative distance to the node $j$ as

$$
\Delta d_{\mathrm{ij}}=\left(\frac{P \delta_{i j}}{\sigma^{2} \Delta \zeta_{i j}}\right)^{1 / 4}
$$

Where $\Delta \zeta$ is the relative signal to noise ratio, it is obtained as

$$
\frac{1}{\Delta \zeta_{i j}}=\left|\frac{1}{\zeta_{j}^{t 2}}-\frac{1}{\zeta_{j}^{t 1}}\right|
$$

and $t_{2}-t_{1}=\alpha$ The relative velocity of the nodes can be given as:

$$
\Delta v_{i j}=\frac{\Delta d_{i j}}{\alpha} m / s e c
$$

Any node in the network transmits the information only after compression (using compressive sensing technique) and Turbo encoding. Let a node $i$ transmits the information $X$, which can be successfully decoded by another node $j \in G_{i}$, where $G_{i}$ be the set of nodes in the transmission region of node $i$. The received information $y_{i}$ at node $j$ is given by [24]: 


$$
y_{j}=\sqrt{P} h_{i j} \tilde{X}+\eta_{j}
$$

Where $h_{i j}$ is the channel coefficient between nodes $i$ and $j$ modeled as complex Gaussian random variable i.e., $h_{i j}=\delta_{i j}^{2} d_{i j}^{-4} ; \delta_{i j}^{2}$ and $d_{i j}$ are the variance and distance between $i$ and $j$; $\tilde{X}$ is the compressed encoded data transmitted by node $i$ and $\eta_{j}$ represents zero mean additive white Gaussian noise with the variance $\sigma^{2}$.

\section{Cluster based D2D cooperative routing}

In this section, we describe the proposed hybrid cluster based D2D cooperative routing scheme for large scale cooperative D2D network in detail. If a source node $N_{S}$ wants to communicate with destination node $N_{d}$, it finds the set of nodes in its transmission coverage region and forms a cluster based on measured metrics (link SNR and relative velocity, as mentioned in system model) using periodically exchanged beacon signals and $N_{s}$ becomes the cluster head. Based on measured metrics, source node determines the cluster and cluster head as $N$, where $N \in G_{N_{S}}$ and is away from the source node. The source node broadcasts the compressed encoded data $\tilde{X}$ along with destination and cluster head ID's.

We denote $N_{n-1}$ as the cluster head of $(n-1)^{t h}$ hop and $G_{n-1}$ as set of nodes of cluster head in $(n-1)^{t h}$ hop. The $n^{t h}$ hop transmission is required only when the destination node is not in the transmission range of $N_{n-1}$. The $n^{\text {th }}$ hop cluster head is selected from the set $\left(G_{n}-G_{n-1}\right) \cup N_{n-1}$ based on sensory measurements:

$$
N_{n}=\underset{l \epsilon\left(G_{n}-G_{n-1}\right) \cup N_{n-1}}{\arg \max }\left\{d_{N_{n-1}, l}\right\} \forall n \geq 2(6)
$$

If the received SNR at the cluster head is less than the predefined threshold (may be because of mobility), i.e., $\varsigma_{N_{h}}<\varsigma_{t h}$, then the cluster head requests the data from its nearest nodes, so that the threshold can be met. The received information at the cluster head is calculated by using MRC technique [11]:

$y_{N_{n}}=h_{N_{n-1}, N_{n}}^{*} y_{N_{n}}+\sum h_{i, N_{n}}^{*} y_{i, N_{n}}$; if $\zeta_{N_{n}}<\zeta_{t h}(7)$

Flow chart for the proposed algorithm is given in Fig. 2.

Algorithm 1:Cluster based D2D cooperative routing

Input: Flow request from source to destination.
Output: Routing path from source to destination with each hop's relay node.

1: $\quad$ while source $\neq$ destination do

2: Source node measures the metrics using periodically exchanged beacon signals.

3: Find set of nodes $\left(G_{N_{S}}\right)$ in its transmission coverage area $R$.

4: If $N_{d} \in G_{N_{s}}$ then

5: cluster head $=$ destination

6: else

7: forms a cluster

$$
V_{h}=\left\{l\left|\left(\max \left(\Delta v_{s i}\right)-\Delta v_{s i}\right)<v_{r ;}\right| l \in\left(G_{n}-G_{n-1}\right) \bigcup N_{n-1}\right\}
$$

8: Based on measurements, source selects cluster head for the next hop as:

$$
N_{n}=\underset{l \epsilon\left(G_{n}-G_{n-1}\right) \cup N_{n-1}}{\arg \max }\left\{d_{N_{n-1}, l}\right\} \forall n \geq 2
$$

9: if $S_{N_{h}}<S_{t h}$ then

10: Cluster head requests the information from nearest nodes.

\section{1: end if}

12: end if

13: source node $=$ cluster head

14: end while

\subsection{Evaluation of energy consumption}

The amount of energy required per bit at $n^{\text {th }}$ hop can be obtained by [24]:

$$
\begin{gathered}
\mathrm{E}_{n-1, n}=(1+\varrho) \zeta_{N_{n}} N_{0} \frac{(4 \pi)^{2} M_{l} \mathcal{N}_{f}}{\mathcal{A}_{N_{n-1}} \mathcal{A}_{N_{n}} \lambda^{2}}\left(d_{N_{n-1}, N_{n}}\right)^{n}+ \\
\frac{P}{\mathcal{R}_{b}}\left(n_{t}+1\right)
\end{gathered}
$$

Where $A_{N_{n-1}}$ and $A_{N_{n}}$ are the gains of transmitter and receiver respectively; $\xi$ is the parameter depends on drain efficiency of power amplifier at the receiver, $\varsigma_{N_{S}}$ is the signal to noise ratio at the node $N_{n}, d_{N_{n-1}, N_{n}}$ is the distance between two successive cluster heads, $M_{l}$ is the link margin, $N_{f}$ is the receiver noise figure, $\lambda$ is the carrier wavelength, $R_{b}$ is the transmission bit rate.

The total energy consumption of path for $H$ hops can be written as

$$
\mathrm{E}=\sum_{i=0}^{H} \mathrm{E}_{\mathrm{i}, \mathrm{i}+1}
$$

\subsection{Evaluation of bit error rate}

The bit error rate (BER) at the $n^{\text {th }}$ hop with $M-$ QAM modulation can be approximated as [25]: 


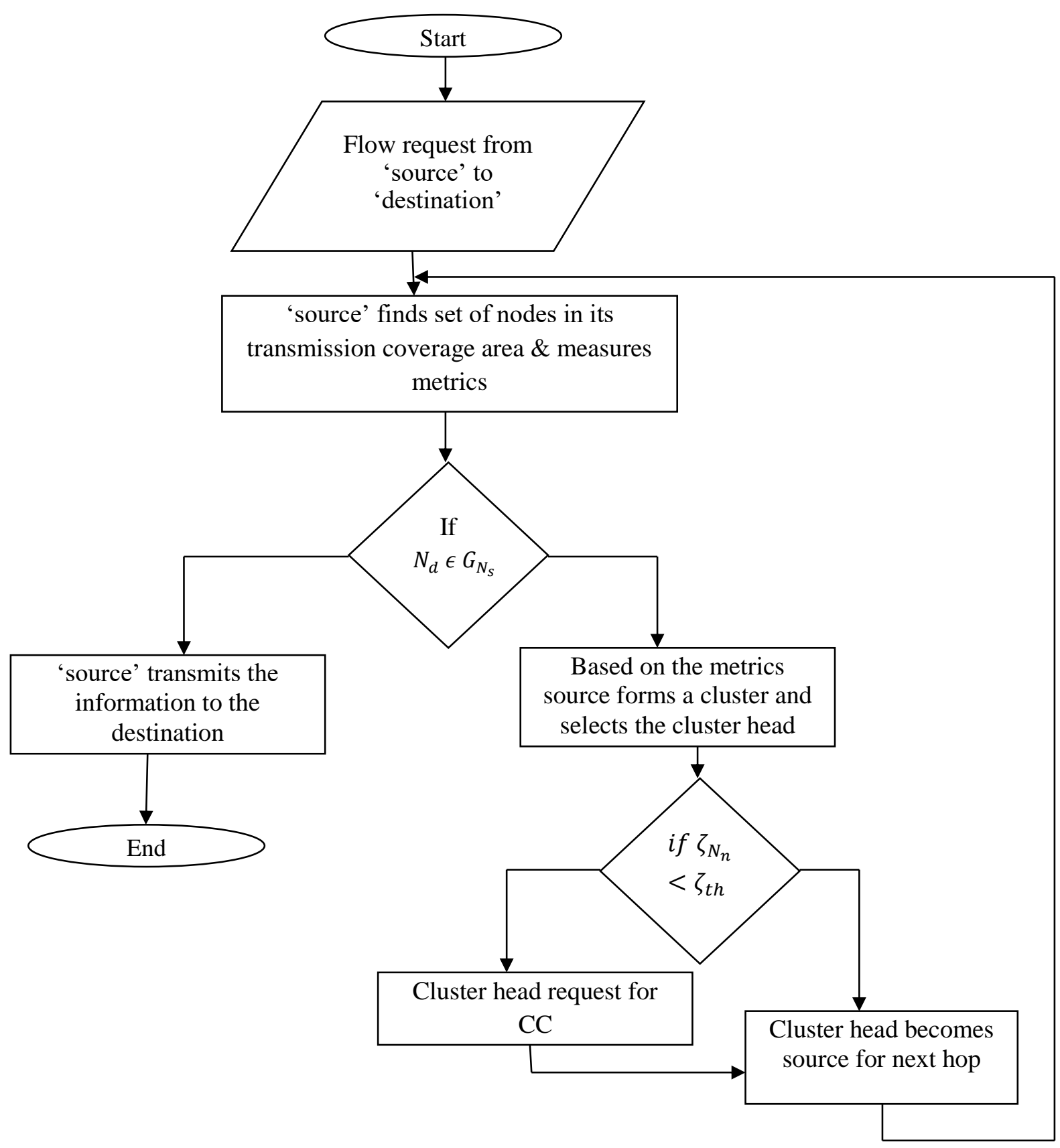

Figure. 2 Flow chart for the proposed routing algorithm

$$
\begin{aligned}
& P_{b}=\frac{2}{\log _{2} \mathcal{M}}\left\{\mathbb{B} Q\left(\frac{P\left(\left|h_{N_{n-1} l}\right|^{2}+\left|h_{l N_{n}}\right|^{2}\right)}{\sqrt{2} \sigma^{2}} \mathbb{D}\right)+\right. \\
& Q\left(\mathbb{D} \frac{\left|h_{N_{n-1} l}\right|}{\sqrt{2} \sigma^{2}}\right)\left[\mathbb{C} Q\left(\mathbb{D} \frac{P\left(\left|h_{N_{n-1} l}\right|^{2}+\left|h_{l N_{n}}\right|^{2}\right)}{\sqrt{2} \sigma^{2}}\right)+\right. \\
& \mathbb{B} Q\left(\mathbb{D} \frac{P\left(\left|h_{N_{n-1} N_{n}}\right|^{2}-\left|h_{l N_{n}}\right|^{2}\right)}{\sqrt{2} \sigma^{2}}\right)+ \\
& \left.\left.\mathbb{B} Q\left(\mathbb{D} \frac{P\left(\left|h_{N_{n-1} l}\right|^{2}+3\left|h_{l N_{n}}\right|^{2}\right)}{\sqrt{2} \sigma^{2}}\right)\right]\right\}
\end{aligned}
$$

Where $\mathbb{B}=\frac{\sqrt{\mathcal{M}}-1}{\sqrt{\mathcal{M}}}$

$$
\begin{aligned}
\mathbb{C} & =\frac{2 \sqrt{\mathcal{M}}-3}{2 \sqrt{\mathcal{M}}} \\
\text { and } \mathbb{D} & =\sqrt{\frac{3}{2(\mathcal{M}-1)}}
\end{aligned}
$$

\section{Simulation results}

Simulation results are presented in this section to show the efficacy of the proposed routing scheme over conventional routing scheme AODV-ETX, CCPT [22] and OCPT [23] routing scheme. The simulation parameters are listed in Table 2 with reference to 3GPP2 Release 12.

Fig. 3 depicts the comparison of three routing schemes with respect to the average number of hops. 
Table 2. Simulation parameters

\begin{tabular}{|l|l|}
\hline Parameter & Value \\
\hline Area & $1000 \times 1000 \mathrm{~m}^{2}$ \\
\hline Number of Nodes & 50 \\
\hline Transmitted Power & $1 \mathrm{~mW}$ \\
\hline SNR Threshold $\left(s_{t h}\right)$ & $3 \mathrm{~dB}$ \\
\hline Noise Variance & $10^{-10}$ \\
\hline Modulation & M-QAM \\
\hline Path loss exponent & 4 \\
\hline Combining Strategy & MRC \\
\hline Periodic interval $(\alpha)$ & $1 \mu \mathrm{s}$ \\
\hline
\end{tabular}

It can be observed that the proposed routing scheme requires less number of hops than AODV-ETX, CCPT and OCPT schemes. Because, as the node density increases in the network, the probability of getting a node away from the source also increases, which forward the information to the destination in minimal path length i.e., in minimum number of hops.

Since the average number of hops decreases, the end-to-end transmission delay and energy consumption of nodes also decreases with an increase in the number of nodes, as shown in Figs. 4 and 5, respectively. Since we are using compressive sensing strategy to compress both the information and beacon signals, the energy consumption of the node is further minimized. The variation of average number of hops, end-to-end transmission delay and energy consumption with respect to the number of nodes in the network are presented in Table 3, Table 4 and Table 5 respectively.

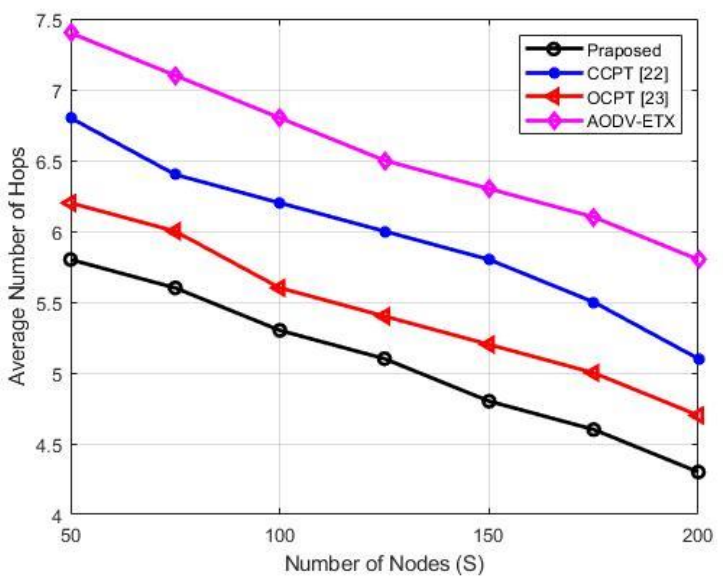

Figure. 3 Average number of hops vs. number of nodes

Table 3. Average number of hops vs number of nodes

\begin{tabular}{|l|l|l|l|l|}
\hline \multirow{2}{*}{$\begin{array}{l}\text { No. of } \\
\text { Nodes }\end{array}$} & \multicolumn{4}{|l|}{ Avg. Number of hops } \\
\cline { 2 - 5 } & Proposed & OCPT & CCPT & AODV \\
\hline 50 & 5.8 & 6.2 & 6.8 & 7.4 \\
\hline 100 & 5.3 & 5.6 & 4 & 6.8 \\
\hline 150 & 4.8 & 5.2 & 6 & 6.3 \\
\hline 200 & 4.3 & 4.7 & 5.5 & 5.8 \\
\hline
\end{tabular}

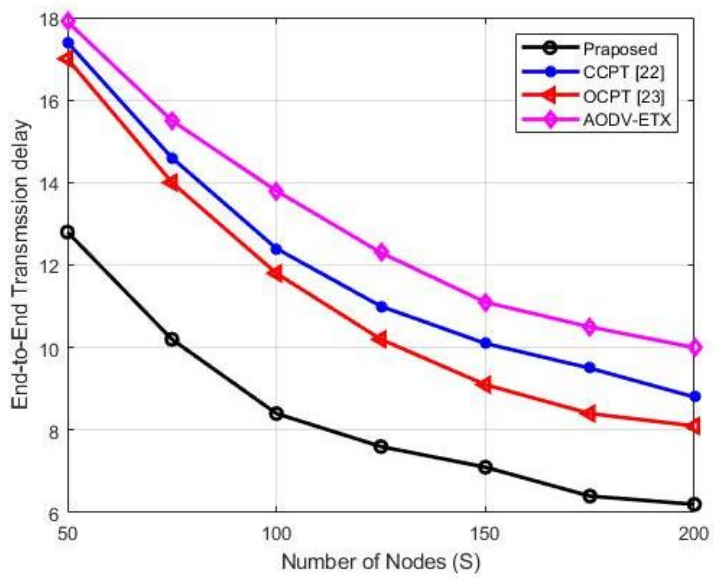

Figure. 4 end-to-end transmission delay vs. number of nodes

Table 4. End-to-end transmission delay vs. number of nodes

\begin{tabular}{|l|l|l|l|l|}
\hline \multirow{2}{*}{$\begin{array}{l}\text { No. of } \\
\text { Nodes }\end{array}$} & \multicolumn{4}{|l|}{ end-to-end transmission delay (ms) } \\
\cline { 2 - 5 } & Proposed & OCPT & CCPT & AODV \\
\hline 50 & 12.8 & 17 & 17.4 & 17.9 \\
\hline 100 & 8.4 & 11.8 & 12.4 & 13.8 \\
\hline 150 & 7.2 & 9.1 & 10.1 & 11.1 \\
\hline 200 & 6.2 & 8.1 & 8.8 & 10 \\
\hline
\end{tabular}

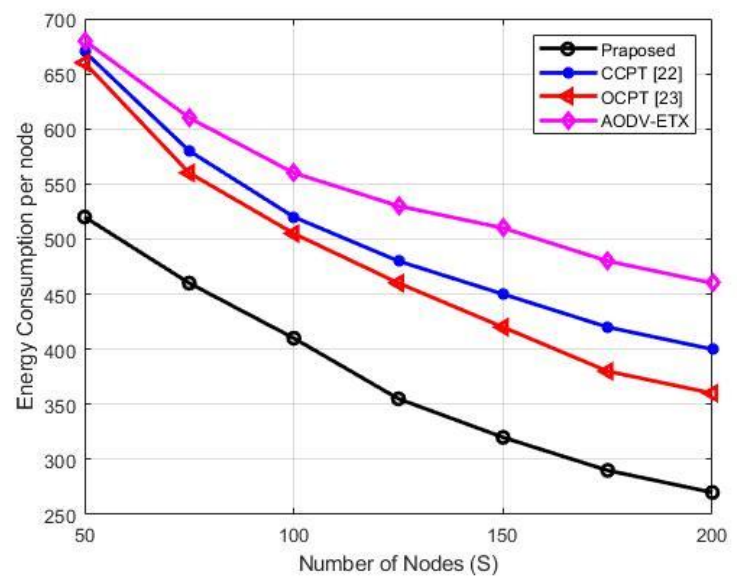

Figure. 5 Energy consumption per node vs. number of nodes

Table 5. Energy consumption per node vs. number of nodes

\begin{tabular}{|l|l|l|l|l|}
\hline \multirow{2}{*}{$\begin{array}{l}\text { No. } \\
\text { Nodes }\end{array}$} & \multicolumn{4}{|l|}{ Energy consumption per node (mJ) } \\
\cline { 2 - 5 } & Proposed & OCPT & CCPT & AODV \\
\hline 50 & 520 & 660 & 670 & 680 \\
\hline 100 & 410 & 505 & 520 & 560 \\
\hline 150 & 320 & 430 & 450 & 510 \\
\hline 200 & 270 & 360 & 400 & 460 \\
\hline
\end{tabular}

The outage probability of the network for a source to destination path is obtained for various routing schemes. The simulated results are presented in Fig. 6 , from the results it can be observed that the proposed routing scheme given better performance than the AODV and OCPT routing schemes. Since 


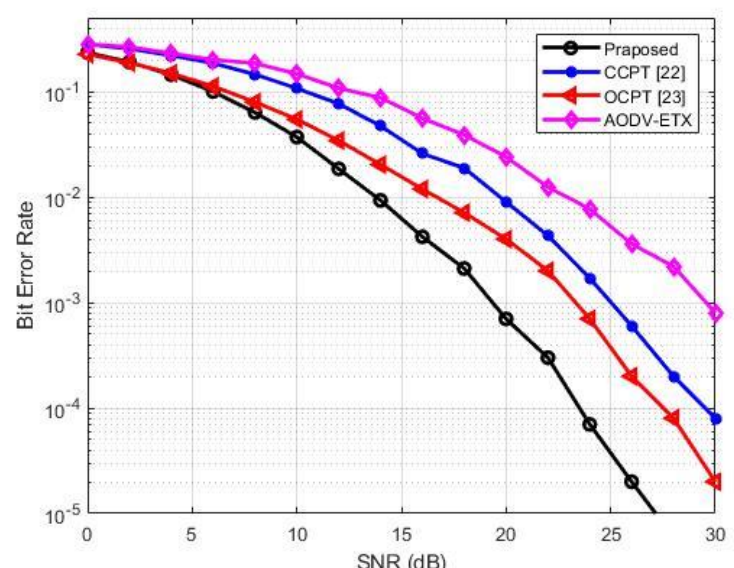

(a)

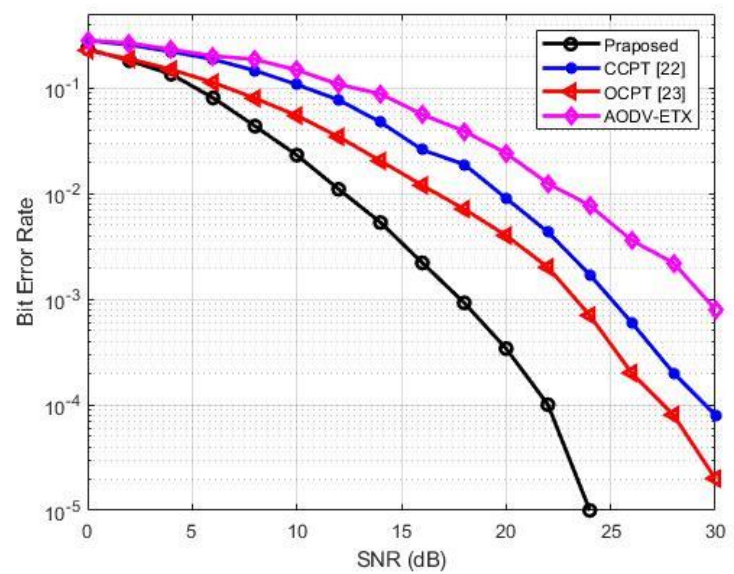

(b)

Figure. 6 BER vs. SNR (dB) with various schemes: (a) $\alpha=50 \mu \mathrm{s}$ and (b) $\alpha=10 \mu \mathrm{s}$

the cluster head requests the information from its nearest nodes whenever the SNR is less than the threshold, the probability of error is minimized. Turbo encoding mechanism also aids in reducing the probability of error. As mentioned in the system model, every node updates the link SNR for every $\alpha$ sec. The dynamic changes in the network can be captured if the nodes update for smaller intervals of time. So, as $\alpha$ decrease, the performance of the proposed algorithm also increases.

\section{Conclusion}

In this paper we proposed and evaluated a hybrid cluster based D2D cooperative communication for large scale networks. This routing scheme is formulated by combining the geographic routing scheme and clustering routing scheme. In this routing, to reduce the impact of mobility on the link reliability, forms a cluster with the nodes having equal (approximately) velocity and based on the location of node we elected cluster head, i.e., the node which is transmission coverage area of source and near to the destination will be act as cluster head for the next hop. Because of mobility and channel conditions if the SNR of at the $\mathrm{CH}$ is less than the pre-defined threshold then the cooperative communication is implemented to improve the network performance. From the simulated results we conclude that, our proposed routing scheme given better performance with respect to the end-to-end transmission delay, energy consumption and outage probability compared to the conventional routing AODV-ETX, Cluster based Cooperative Packet Transmission (CCPT) and opportunistic cooperative packet transmission (OCPT) scheme. Proposed algorithm improves the end-to-end transmission delay by $39 \%$ (approximately) and energy consumption by $35 \%$ compared to AODV algorithm. Future work will be carried out research by considering effect of imperfect synchronization.

\section{Conflicts of Interest}

The authors declare no conflict of interest.

\section{Author Contributions}

The paper conceptualization, methodology, software, validation, formal analysis, investigation, resources, data curation, writing-original draft preparation, writing-review editing and visualization, have been done by $1^{\text {st }}$ author. The supervision and project administration have been done by $2^{\text {nd }}$ and $3^{\text {rd }}$ authors.

\section{References}

[1] A. Nosratinia, T. E. Hunter, and A. Hedayat, "Cooperative communication in wireless networks", IEEE Communications Magazine, Vol. 42, No. 10, pp. 74-80, 2004.

[2] D. P. Kumar, M. S. Babu, P. P. Saradhi, and M. G. Krishna, "Distribute coding for OFDM based cooperative HF radio communication system", International Journal of Recent Technology and Engineering, Vol. 8, No. 2, pp. 3130-3142, 2019.

[3] Y. Cao, T. Jiang, and C. Wang, "Cooperative device-to-device communications in cellular networks", IEEE Wireless Communications, Vol. 22, No. 3, pp. 124-129, 2015.

[4] M. N. Tehrani, M. Uysal, and H. Yanikomeroglu, "Device - to - device communication in 5G cellular networks: challenges, solutions, and future directions", IEEE Communications Magazine, Vol. 52, No. 5, pp. 86-92, 2014.

[5] Y. C. Tseng, W. H. Liao, and S. L. Wu, "Mobile ad hoc networks and routing protocols", John 
Wiley \& Sons, Inc., Hoboken NJ, pp. 371-391, 2012.

[6] N. Srikanth and M.S.G. Prasad, "Family based Efficient Routing Protocol (FERP) for lifetime improvement in heterogeneous wireless sensor networks", Journal of Advanced Research in Dynamical and Control Systems, Vol. 11, No. 6, pp.131-140, 2019.

[7] S. M. Zin, N. B. Anuar, L. M. Kiah, and A. K. Pathan, "Routing protocol design for secure WSN: Review and open research issues", Journal of Network and Computer Applications, Vol. 41, No. 1, pp. 517-530, 2014.

[8] R. Sadakale, R. A. Patil, and N. V. K. Ramesh, "An efficient AODV routing protocol for vehicular Ad hoc network", International Journal of Innovative Technology and Exploring Engineering, Vol. 8, No. 4, pp.1-4, 2019.

[9] C. R. Prasad and P. Bojja, "A survey on routing protocols in wireless body area networks for medical applications", Journal of Advanced Research in Dynamical and Control Systems, Vol. 10, No. 10, pp. 92- 97, 2018.

[10]N. Nasser, C. Arboleda, and M. Liliana, "Comparison of Clustering Algorithms and Protocols for Wireless Sensor Networks", In: Proc. of Canadian Conf. on Electrical and Computer Engineering, Ottawa, Ont., pp. 17871792, 2006.

[11]P. S. Reddy, P. S. Akram, M. A. Sharma, P. A. S. Ram, and R. P. Raj, "Study and analysis of routing protocols", International Journal of Emerging Trends in Engineering Research, Vol. 7, No. 11, pp.434-440, 2019.

[12]C. Cynthia, P. K. Saguturu, K. Bandi, S. Magulluri, and T. Anusha, "A survey on MANET protocols in wireless sensor networks", International Journal of Engineering and Technology, Vol. 7, No. 2, pp. 1-3, 2018.

[13]N. Sabor and M. Abo-Zahhad, "A Comprehensive Survey of Intelligent-Based Hierarchical Routing Protocols for Wireless Sensor Networks", Nature-Inspired Computing for Wireless Sensor Networks, Springer, Singapore, pp. 197-257, 2020.

[14]S. Shafi, and D. V. Ratnam, "A Cross Layer Cluster Based Routing Approach for Efficient Multimedia Data Dissemination with Improved Reliability in VANETs", Wireless Personal Communications, Vol. 107, No. 4, pp.21732190, 2019.

[15]L. Bin, W. J. Wang, Q Y Yin, H. X. Li and R. Yang, "An energy efficient geographic routing based on cooperative transmission in wireless sensor networks", Science China Information Sciences, Vol. 56, No. 7, pp. 4757- 4762, 2013.

[16]S. Syue, C. Wang, T. Aguilar, V. Gauthier and H. Afifi, "Cooperative Geographic Routing with Radio Coverage Extension for SERConstrained Wireless Relay Networks", IEEE Journal on Selected Areas in Communications, Vol. 30, No. 2, pp. $271-279,2012$.

[17]L. Hung, F. Leu, K. L. Tsai, and C. Y. Ko, "Energy-Efficient Cooperative Routing Scheme for Heterogeneous Wireless Sensor Networks", IEEE Access, Vol. 8, No. 1, pp. 56321-56332, 2020.

[18]M. Asshad, A. Kavak, K. Kucuk, and S. A. Khan, "Using Moment Generating Function for Performance Analysis in NonRegenerative Cooperative Relay Networks with Max-Min Relay Selection", $A E U$ International Journal of Electronics and Communications, Vol. 116, No. 1, pp. 1-10, 2020.

[19]B. Lv, R. Wang, Y. Cui, Y. Gong, and H. Tan, "Joint Optimization of File Placement and Delivery in Cache-Assisted Wireless Networks with Limited Lifetime and Cache Space", IEEE Transactions on Communications, Vol. 68, No. 4, pp. 23392354, 2020.

[20]C. Perkins, E. B. Royer, and S. Das, "Ad Hoc On-Demand Distance Vector (AODV) Routing", Internet Requests for Comments, RFC Editor, RFC 3561. 2003. Available online: https://www.ietf.org/rfc/ rfc3561.txt (accessed on 15112019 ).

[21]H. D. Trung, W. Benjapolakul, and P. M. Duc, "Performance evaluation and comparison of different ad hoc routing protocols", Computer Communications, Vol. 30, No. 11-12, pp. 24782496, 2007.

[22]J. Cheng, Y. Gao, L. Yan, and H. Yang, “A novel cluster-based cooperative packet transmission protocol for wireless ad-hoc networks", IEICE Transactions on Communications, Vol. E102-B, No. 4, pp. 768 778, 2019.

[23]Y. Gao, N. Zhang, J. Cheng, and G. Kang, “An Opportunistic Cooperative Packet Transmission Scheme in Wireless Multi-Hop Networks", In: Proc. of MDPI, Sensors, IEEE Wireless Communications and Networking Conf. Workshop (WCNCW), Marrakech, Morocco, pp. 1-17, April 2019. 
[24]D. P. Kumar, P. P. Saradhi, and M. S. Babu, "Energy Efficient Transmission for MultiRadio Multi-Hop Cooperative Wireless Networks", Journal of Advanced Research in Dynamical and Control Systems, Vol. 10, No. 4, pp. 117-122, 2018.

[25]D. P. Kumar, P. P. Saradhi, and M. S. Babu, "Large Scale Cooperative Wireless Networks: Relay Selection and Performance Analysis", International Journal of Advanced Trends in Computer Science and Engineering, Vol. 9, No. 4, pp. 6400-6405, 2020. 